Abstract

\title{
Development of Zika Virus DNA Vaccine Using Envelope Modified Baculoviral Gene Delivery System $^{+}$
}

\author{
Hanul Choi ${ }^{1}$, Yuyeon Jang ${ }^{1}$, Hansam Cho ${ }^{2}$, Ha Youn Shin ${ }^{1}$ and Young Bong Kim ${ }^{1, *}$ \\ 1 Department of Biomedical Science and Engineering, University of Konkuk, Seoul 05029, Korea; \\ chlgksmf9977@hanmail.net (H.C.); wkddbfkd@naver.com (Y.J.); hayounshin@konkuk.ac.kr (H.Y.S.) \\ 2 KRBIOTECH. Co. LTD, Kwangjin-gu, Seoul 05029, Korea; polomaniaye@hanmail.net \\ * Correspondence: kimera@konkuk.ac.kr \\ + Presented at Viruses 2020-Novel Concepts in Virology, Barcelona, Spain, 5-7 February 2020.
}

Published: 17 June 2020

\begin{abstract}
Zika virus (ZIKV) is a mosquito-borne flavivirus and the infection of pregnant women can cause a wide range of congenital abnormalities, including microcephaly in the infant. However, there is no vaccine available yet. In this study, we intended to use $\operatorname{PrM} / \mathrm{E}$, which is the main target gene of neutralizing antibodies, for the development of a DNA vaccine for ZIKV. To enhance the gene delivery, a recombinant baculovirus whose surface was modified to express human endogenous retrovirus (HERV) envelope was constructed. Baculovirus with HERV envelope (AcHERV) showed distinguished higher gene delivery than wild type. Using the AcHERV as a delivery vector, we constructed major antigen (prM-E)-encoding DNA under the CMV promoter, AcHERV-ZIKA. Transducing of prM/E gene in a mammalian cell was confirmed by Western blot. Immunization in mice with 10e7 of AcHERV-ZIKA elicited high IgG and neutralizing antibodies. In the challenge test, AcHERV-ZIKA immunized A129 mice showed perfect protection. These results suggest that AcHERV-ZIKA could be a potential vaccine candidate for human application.
\end{abstract}

Keywords: Zika virus; DNA vaccine; baculovirus

(C) 2020 by the authors. Licensee MDPI, Basel, Switzerland. This article is an open access article distributed under the terms and conditions of the Creative Commons Attribution (CC BY) license (http://creativecommons.org/licenses/by/4.0/). 